\title{
A Smart Energy Meter Enabling Self-Demand Response of Consumers in Smart Cities of Tamil Nadu
}

\author{
Shashank Singh \\ Hybrid Electrical Systems Lab, Dept. of EEE \\ National Institute of Technology Tiruchirappalli \\ Tiruchirappalli, India \\ shashanksingh0110@gmail.com
}

\author{
M. P. Selvan \\ Hybrid Electrical Systems Lab, Dept. of EEE \\ National Institute of Technology Tiruchirappalli \\ Tiruchirappalli, India \\ selvanmp@nitt.edu
}

\begin{abstract}
Demand response (DR) is one of the demand side management features under the paradigm of smart grids, wherein the consumers are encouraged to participate in the utility operations through active response to electricity price signals by altering their demand patterns. One impediment in employing demand response schemes in India is the fixed electricity tariff for the domestic consumers, which most of the Indian state utilities follow. Interestingly, the Tamil Nadu State Electricity Board (TNEB) follows an incremental block rate tariff for domestic consumers, which provides an opportunity for the implementation of proposed self-DR scheme. Hence firstly, this paper presents the design and development of a low-cost singlephase smart energy meter (SEM) which incorporates a TNEB tariff structure. Secondly, the development of an indigenous meter data management system (MDMS) software is attempted for the utility using open-source software tools. Finally, the idea of self-DR is introduced and emphasized through the coordinated operation of developed SEM and MDMS.
\end{abstract}

Keywords - demand response, meter data management system, smart cities, smart energy meter, smart grids

\section{INTRODUCTION}

The electric power entity is an important sector to be digitized under the Indian Smart Cities mission. This transition will lead to a smarter grid envisioning a consumer-facing grid capable of overcoming key issues such as ever increasing electricity demand, depletion of conventional energy resources, and stable penetration of renewable energy resources [1]. Considering the growing imbalance between generation and demand, sustainable power sources are becoming the need of the hour. However, the factors such as presence of harmonics in voltage and current signals, and intermittent nature of power production entails the complexity in the management of energy flow. This implies that real-time sensing, monitoring, control, and demand management technologies will play a major role in the future of power grids or smart grids (SG) [2].

Demand response (DR) is one of the key demand side management features of SG environment, intended towards

This publication is an outcome of the R\&D work undertaken in the project under the Visvesvaraya PhD Scheme of Ministry of Electronics and Information Technology, Government of India, being implemented by Digital India Corporation (formerly Media Lab Asia). healthy operation of grid by offering financial incentives or demand based tariffs to consumers upon reduction of their peak load demand [3], [4]. The smart energy meters (SEM) could become crucial elements to facilitate DR among consumers in SG environment.

The application and development of SEM(s) are observed to be progressive and continuous in recent years. Gungur et al. [5] have surveyed the diverse range of electronics and communication technologies related to SEM. References [6]-[10] have discussed architecture, communication technologies, the web and data service aspects, and a framework for data privacy for the deployment of smart meters. The challenges in future applications of SEM and its data analytics are comprehensively analyzed, and smart metering has been referred to as the future way of life in [11].

The development of Arduino based SEM integrated with GSM and GPRS are proposed in [12]-[16]. Following the advancement in meter developments, a non-contact based meter was proposed in [17]. SG has been referred to as an intelligent energy system in [18], which has reviewed SEM along with smart gas and heat meters. A power meter with consumer side load management was attempted in [19]. Three-phase smart sensors for SG applications are proposed in [20], [21] with emphasis on accuracy of estimation of key parameters. Furthermore, a review on sensing technologies and role of IoT for next generation power grids are given in [22].

The key difference between existing literature and this meter is its data-analytic, bill prediction feature, the development of its data management software (MDMS) using open-source tools, and low-cost. The novel aspects of the system are as follows:

- The SEM is capable of measuring sinusoidal and nonsinusoidal load currents.

- The SEM is capable of implementing any tariff structure (i.e. block rate, fixed rate, etc.).

- The SEM computes energy consumption and estimates consumption pattern (rate of consumption for current month), predicted consumption and electricity bill.

(C) 2019 IEEE. Personal use of this material is permitted. Permission from IEEE must be obtained for all other uses, in any current or future media, including reprinting/republishing this material for advertising or promotional purposes, creating new collective works, for resale or redistribution to servers or lists, or reuse of any copyrighted component of this work in other works 
- The SEM has a GSM module which notifies consumers about their consumption patterns and predicted bill via SMS.

- The SEM is capable of transmitting data to utility's server over the Internet.

- The MDMS has been developed using open-source software tools.

- The developed component of MDMS provides a private user interface to the consumer.

- Introduction of the concept of Self-DR and its integration with MDMS

The rest of this paper is organized in the following way: section II deals with the design, testing, and features of SEM; the development of MDMS is detailed in section III; section IV introduces self-DR, and section $\mathrm{V}$ concludes the paper.

\section{Design and Features of Smart Energy Meter}

A successful implementation of metering technology needs an analog data acquisition and processing unit. Major constituents of the proposed meter are ATmega2560 microcontroller, voltage and current signal conditioning units (VCU and CCU), a real-time clock (RTC), WiFi and GSM based communication modules, and an organic light emitting diode (OLED) display unit. The block diagram and hardware prototype of the proposed single-phase SEM are shown in Fig. 1.

The technical specifications of meter are reported in Table I. The energy measurement principles, real time acquisition of voltage and current to compute electrical parameters of load, testing of transducers, communication systems, the role of peripherals, and features are explained in the forthcoming subsections.

TABLE I

SEM SPECIFICATIONS

\begin{tabular}{cc}
\hline \hline Parameters & Range \\
\hline Voltage $(\mathrm{rms})$ & $0-300 \mathrm{~V}$ \\
Current $(\mathrm{rms})$ & $0-30 \mathrm{~A}$ \\
ADC Resolution & $10 \mathrm{bit}$ \\
Supply Voltage $(\mathrm{DC})$ & $12 \mathrm{~V}$ \\
\hline \hline
\end{tabular}

\section{A. Fundamental Background of Energy Measurement}

An energy meter measures the amount of electrical energy consumed by the consumer expressed in kilowatt hour $(k W h)$. The electrical power triangle comprises of active power $(P)$, reactive power $(Q)$, and apparent power $(S)$. Energy $(E)$ is the accumulation of active power over a period of time given by (1), where $T$ represents the period of time.

$$
E=\int_{0}^{T} P(t) d t
$$

The direct execution of (1) is not possible in microcontroller. The sampling and processing of analog signals require preamble of analog to digital converter (ADC), which provides discrete value of voltage and current signals. The measurement of $P$ requires discrete samples of voltage and current signals

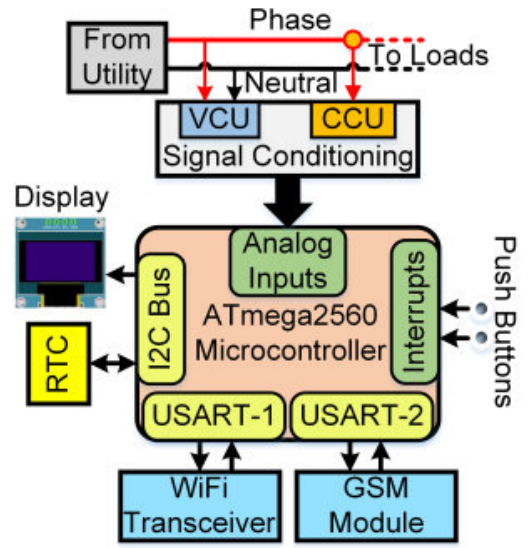

(a) Block diagram of SEM

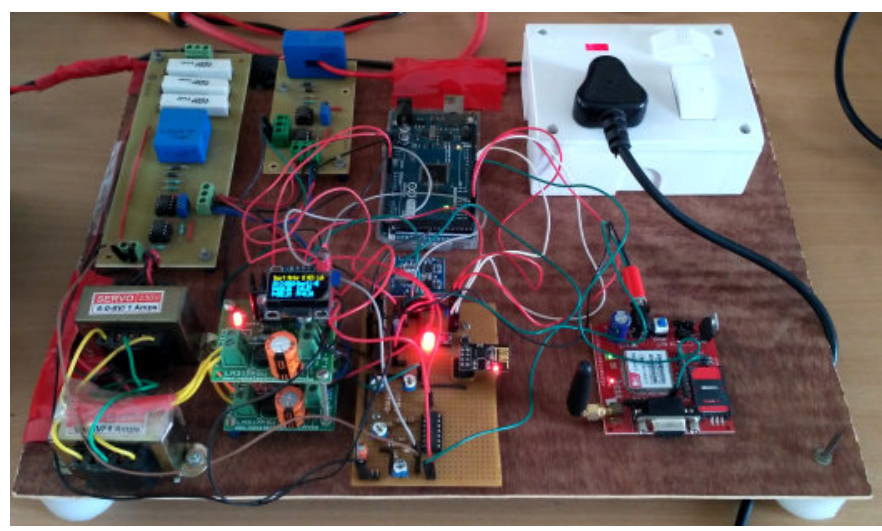

(b) Hardware prototype of SEM developed in laboratory

Fig. 1. Proposed single-phase smart energy meter for self-DR

that are acquired using developed signal conditioning circuits. The samples acquired from VCU and CCU are processed through two channels of an ADC of microcontroller, which discretizes the analog samples for further computation. The $P$ can be written as a function of voltage $(V)$ and current $(I)$ signals using (2). Furthermore, the $r m s$ value of a signal $(\lambda)$ is defined as (3), where $\lambda \in\{V, I\} ; N$ represents total number of samples in one fundamental period (i.e. 20 milliseconds for $50 \mathrm{~Hz}$ signal), and $n$ represents sample at $n^{t h}$ instant. The multiplication of $V_{r m s}$ and $I_{r m s}$ provides the value of $S$ (4). In a practical scenario, the nature of load current depends on the type of load: it cannot always be assumed as pure sinusoidal for all household appliances. This implies that the cosine of the phase angle difference between $V$ and $I$ will not represent the true power factor $(P F)$ of the load, rather it is defined as the ratio of $P$ consumed by the load to $S$ supplied to it (5).

$$
\begin{gathered}
P=\frac{1}{N} \sum_{n=1}^{N} V[n] \times I[n] \\
\lambda_{r m s}=\sqrt{\frac{1}{N} \times \sum_{n=1}^{N}(\lambda[n])^{2}} \\
S=V_{r m s} \times I_{r m s}
\end{gathered}
$$




$$
P F=\frac{P}{S}
$$

\section{B. Voltage and Current Conditioning Units}

The schematic of signal conditioning circuit and its connection with ADC of microcontroller are depicted in Fig. 2. An industry grade voltage sensor (VS), LV20-P (connected across single-phase AC power supply through $30 \Omega, 10 \mathrm{~W}$ current limiting resistor) produces an output current $\left(I_{R_{m 1}}\right)$ if a chosen value of resistor $R_{m 1}(100 \Omega)$ is connected on its measurement pin. The resultant voltage drop across $R_{m 1}$ ( $I_{R_{m 1}} R_{m 1}$, which is proportional to the single-phase $\mathrm{AC}$ voltage $v(t)$ ) is amplified using pair of operational amplifiers having net gain $A_{v 1}$. Similarly, an industry grade current sensor (CS), LA55-P is used to sense the supply current $i(t)$, which also produces an output current $\left(I_{R_{m 2}}\right)$ if a chosen value of resistor $R_{m 2}(100 \Omega)$ is connected on its measurement pin. The resultant voltage drop across $R_{m 2}\left(I_{R_{m 2}} R_{m 2}\right.$, that is proportional to the supply current $i(t)$ passing through CS) is amplified using another pair of operational amplifiers having net gain $A_{i 1}$. Due to the unipolar nature of $\mathrm{ADC}$ of micrcontroller, a DC bias voltage of $V_{D C}$ is introduced separately through two summing amplifiers having gains $A_{v 2}$ and $A_{i 2}$ which yield net output voltages namely $v_{v}(t)$ and $v_{i}(t)$. These output voltage are then applied on two channels of the ADC of microcontroller for further processing.

The further processing and calculation of parameters mentioned in (2)-(5) from the acquired samples require filtering of added DC offset voltage. There are two approaches suggested by [19] for filtering the DC bias voltage. This work suggests a third approach; the application of Fourier series. The steps involved in filtering operations are expressed through (6).

$$
\left[\begin{array}{c}
V[n] \\
I[n]
\end{array}\right]=\left[\begin{array}{cc}
S_{v}{ }^{-1} & 0 \\
0 & S_{i}^{-1}
\end{array}\right] \times\left[\begin{array}{l}
v_{v}[n]-\frac{1}{N} \sum_{n=1}^{N} v_{v}[n] \\
v_{i}[n]-\frac{1}{N} \sum_{n=1}^{N} v_{i}[n]
\end{array}\right]
$$

where, $\left(v_{v}(t), v_{i}(t)\right) \stackrel{\text { sampling }}{\longrightarrow}\left(v_{v}[n], v_{i}[n]\right)$ $S_{v} \Rightarrow$ sensitivity of VS in $m V / V$ and, $S_{v}=\frac{v_{v}(t)}{v(t)}=\frac{\left(I_{R_{m 1}} R_{m 1} A_{v 1}+V_{D C}\right) A_{v 2}}{v(t)}$

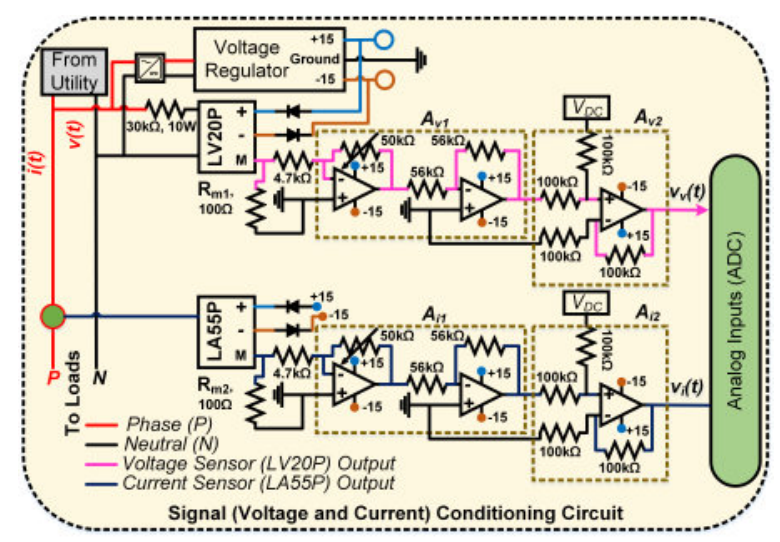

Fig. 2. Interfacing of VS and CS with ADC of micrcontroller
$S_{i} \Rightarrow$ sensitivity of CS in $m V / A$ and, $S_{i}=\frac{v_{i}(t)}{i(t)}=\frac{\left(I_{R_{m 2}} R_{m 2} A_{i 1}+V_{D C}\right) A_{i 2}}{i(t)}$

\section{Experimental Testing of SEM}

The performance of SEM measurements is evaluated using Fluke 434 (series II) energy analyzer (EA), Agilent $1146 \mathrm{~B}$ AC/DC current probe with Agilent InfiniiVision DSOX 2014A digital storage oscilloscope (DSO). Two standard instruments (EA and Agilent current probe) have been used to evaluate the accuracy of $I_{r m s}$ measurement, whereas only EA is used to evaluate the accuracy of $V_{r m s}, P$, and $P F$ measurements. Further, six combinations of loads are connected to SEM for testing, and the loads are referred here as load-1, load-2,..., load-6. The description of those loads are given in Table. II. The current waveform of each combination of load is observed using DSO and illustrated in Fig. 3, that justifies the participation of loads having high current harmonics in the tests. The measured value of current is compared with aforementioned standard instruments in Fig. 4. The measured values of $P, V_{r m s}$ and $P F$ are compared with the aforementioned standard instruments and illustrated in Fig. 5. From the experimental results, it can be observed that the measurement accuracy of the developed SEM is comparable with the standard instruments.

TABLE II

DESCRIPTION OF PARTICIPATING LOADS

\begin{tabular}{ccc}
\hline \hline Reference & Name & Details \\
\hline Load-1 & NL Load & 230V, 0.53A \\
Load-2 & RL Load & Auto-Transformer + Rheostat \\
Load-3 & RL + NL Load & Load $(1+2)$ \\
Load-4 & Blower & $230 V, 600 \mathrm{~W}$ \\
Load-5 & Blower + NL Load & Load $(1+5)$ \\
Load-6 & RL + Blower + NL Load & Load $(1+2+4)$ \\
\hline \hline
\end{tabular}

NL: Non-linear, Rheostat: $9 \Omega$ 10A, RL: Combination of R \& L type load

\section{Communication Unit, Peripherals \& Features}

The communication module includes a WiFi transceiver and a GSM module, which are interfaced with SEM on its microcontroller's USART ports. The role of the WiFi transceiver is to exchange data with the utility's server and in-home display, whereas the role of GSM module is to notify the rate of energy consumption, current bill and predicted bill for that month to the consumer via SMS.

The associated peripherals are an OLED display, two pushbuttons, and a RTC. The OLED display is the primary display of the meter that displays values of electrical parameters described earlier (see II-A). The integrated RTC overcomes microcontroller's internal timer overflow problem and in turn yields accurate timing signals for the computation of energy consumption. The OLED display and RTC are connected on $\mathrm{I}^{2} \mathrm{C}$ (inter-integrated circuit) bus of microcontroller.

The push-buttons generate interrupt to temporarily display values of current billing tariff, total energy consumption, rate of energy consumption, and predicted energy consumption for current month on primary display. The estimation of 

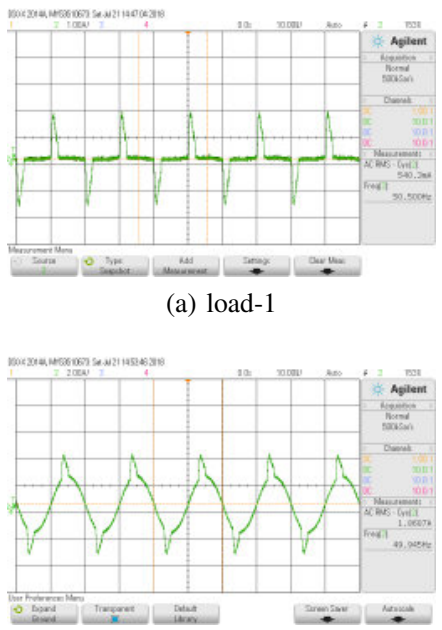

(c) load-3

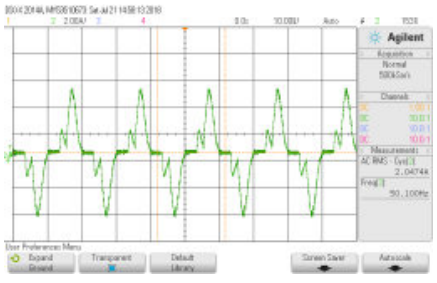

(e) load-5 (a) load-1

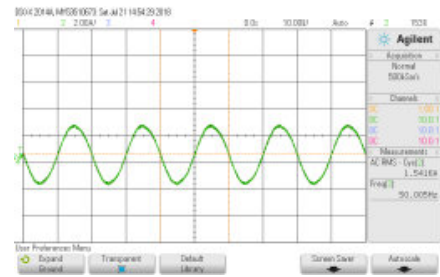

(b) load-2

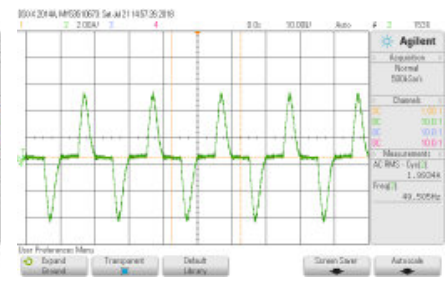

(d) load-4

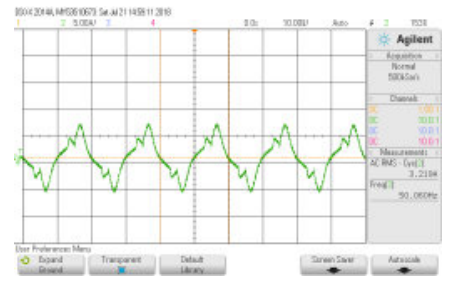

(f) load-6
Fig. 3. Current waveform for different types of loads

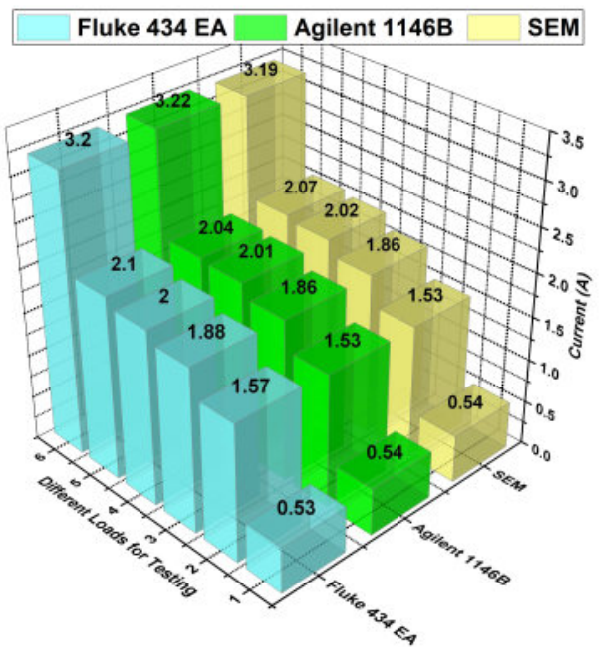

Fig. 4. Performance evaluation of current sensing circuit

aforementioned display parameters are key features of this SEM. The main display panel and navigation through on request temporary displays are depicted in Fig. 6, wherein the displayed unit of energy consumption has been scaled down to $W h$ for laboratory experiment purposes.

\section{Design of Meter Data Management System}

The proposed MDMS has two consumer oriented display components: the first being an in-home display and the second
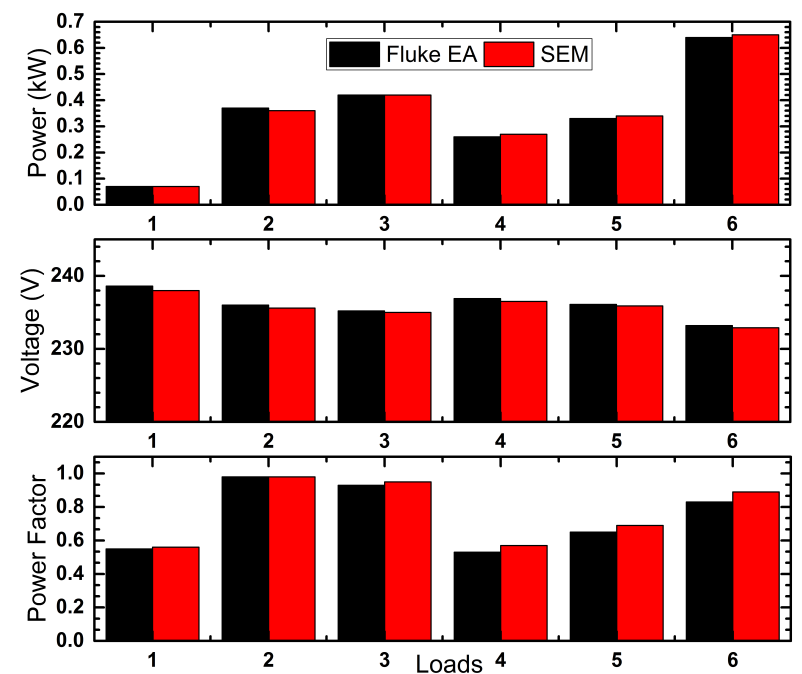

Fig. 5. Measurement of active power, voltage and power factor

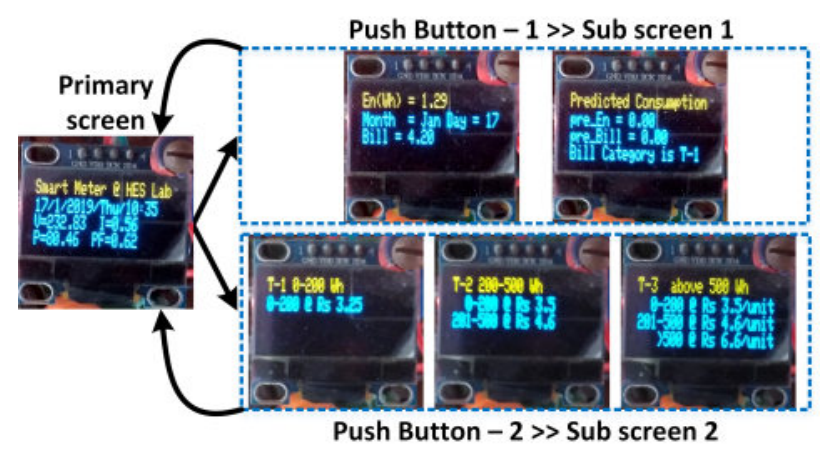

Fig. 6. Illustration of display panel of SEM

being the web based framework on utility's server. This paper deals with the development of in-home display component of MDMS, whereas the web based framework on utility's server is under testing and constitutes the future of presented work. The development of in-home display component was done using Python. Further, the library level dependencies are Tkinter GUI package and SQLite database engine. The block diagram depicting exchange of data with utility and in-home display is shown in Fig. 7. It is evident from the illustration that the meter directly communicates with utility's server though internet for the exchange of key parameters, whereas it exchanges data with a load management unit (LMU) [4] to show it on the in-home display. The LMU exchanges data with meter via serial communication and stores it in a database (server.py), and an another program (GUI.py) fetches the data from that database and displays it via a graphical user interface.

\section{Self-Demand Response Scheme}

The idea of self-DR is inspired from an initiative taken by Southern California Edison Power Station to reduce the demand of electricity by introducing Energy Orb [23]. 


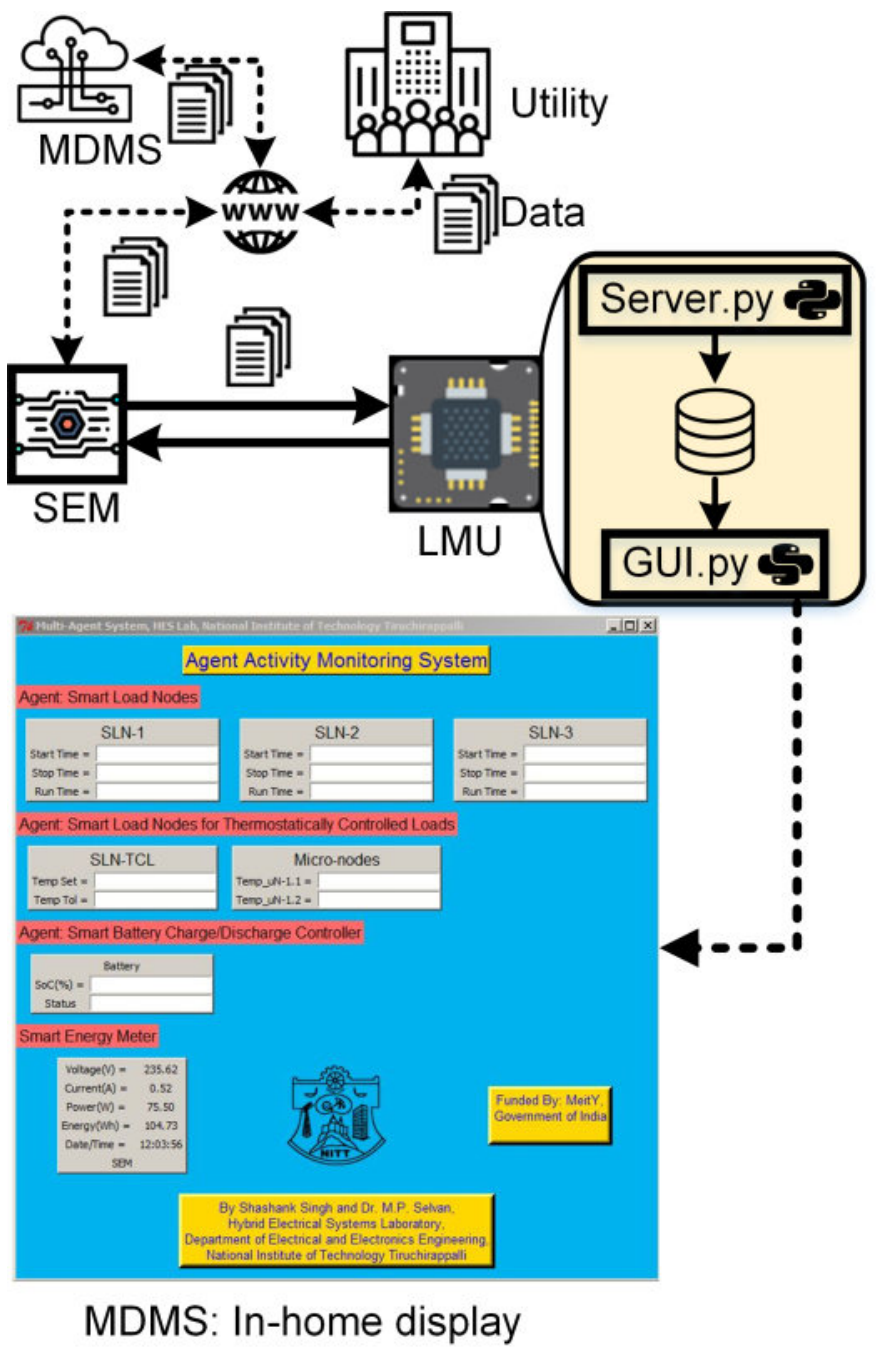

Fig. 7. Communication model of SEM with the utility and LMU

Energy Orb is a glowing sphere that changes its color to indicate low and high prices of electricity. It reportedly brought a $40 \%$ reduction in energy usage for consumers using it. It has proved to be a non-intrusive approach persuading consumers to change their consumption patterns according to the color of the Orb. There are other similar devices introduced in the past which are mentioned in [23], [24]. Furthermore, another nonverbal approach named ColorPower proposed by an MIT startup gives color based indications to consumers alerting them to exert a control on power consumption during peak hours [25] [26].

In this paper, the SEM incorporates a flexible tariff for energy billing which is referred to as incremental blockrate tariff (IBRT) structure. IBRT structure is adopted from Tamil Nadu state electricity board's low tension supply tariff [27]. The notification through the SMS of daily energy consumption, current bill, the rate of consumption, and predicted bill according to aforementioned IBRT could encourage the consumers to alter their consumption patterns in order to reduce the monthly electricity bill. This is referred in here as self-DR, the most suitable one for the smart cities in Tamil Nadu state and claimed to be a feature of this presented system.

\section{CONCLUSION}

The proposed meter and its data management system creates a motivating and interactive environment for the consumers, wherein diverse data visualization methods, and persuasive interfaces allow them to explore their consumption patterns in a simple and straightforward way. The key elements: a lowcost microcontroller based single-phase smart energy meter for self-demand response using price prediction and in-home display component of meter data management system have been practically implemented in the laboratory using opensource software frameworks.

It is evident from the presented experimental description that the energy meter unit measures consumers' electrical parameters by processing the voltage and current signals of different type of loads. Some of the processed information is available to the consumers through organic light emitting diode display in the meter and short messaging service. Moreover some are also available on developed in-home display, and on utility's server.

The presented work is an attempt towards the design and development of advanced metering infrastructure enabled with nonintrusive load monitoring scheme and agent based system for residential demand response using indigenous opensource/free tools and framework for future smart grid of India.

\section{REFERENCES}

[1] S. E. Collier, "The emerging enernet: Convergence of the smart grid with the Internet of Things," IEEE Industry Applications Magazine, vol. 23, no. 2, pp. 12-16, 2017.

[2] R. Morello, C. De Capua, G. Fulco, and S. C. Mukhopadhyay, "A smart power meter to monitor energy flow in smart grids: The role of advanced sensing and IoT in the electric grid of the future," IEEE Sensors Journal, vol. 17, no. 23, pp. 7828-7837, 2017.

[3] P. Palensky and D. Dietrich, "Demand side management: demand response, intelligent energy systems, and smart loads," IEEE Transactions on Industrial Informatics, vol. 7, no. 3, pp. 381-388, 2011.

[4] S. Singh, A. Roy, and M. P. Selvan, "Smart load node for nonsmart load under smart grid paradigm: A new home energy management system," IEEE Consumer Electronics Magazine, vol. 8, no. 2, pp. 22-27, March 2019.

[5] V. C. Gungor, D. Sahin, T. Kocak, S. Ergut, C. Buccella, C. Cecati, and G. P. Hancke, "Smart grid technologies: communication technologies and standards," IEEE Transactions on Industrial informatics, vol. 7, no. 4, pp. 529-539, 2011

[6] P. Prudhvi, D. Bhalodi, M. Manohar, V. Padidela, and S. Adapa, "A smart energy meter architecture in Indian context," in Proc. 2012 11th International Conference on Environment and Electrical Engineering (EEEIC), Tehran, Iran, pp. 217-222.

[7] J. Zheng, D. W. Gao, and L. Lin, "Smart meters in smart grid: an overview," in Proc. 2013 IEEE Green Technologies Conference, Denver, CO, USA, pp. 57-64.

[8] S. Prasad and S. Avinash, "Smart meter data analytics using OpenTSDB and Hadoop," in Proc. 2013 IEEE Innovative Smart Grid TechnologiesAsia (ISGT Asia), Bangalore, India, pp. 1-6.

[9] L. Sankar, S. R. Rajagopalan, and S. Mohajer, "Smart meter privacy: a theoretical framework," IEEE Transactions on Smart Grid, vol. 4, no. 2, pp. 837-846, 2013.

[10] L. Yang, X. Chen, J. Zhang, and H. V. Poor, "Cost-effective and privacypreserving energy management for smart meters," IEEE Transactions on Smart Grid, vol. 6, no. 1, pp. 486-495, 2015. 
[11] D. Alahakoon and X. Yu, "Smart electricity meter data intelligence for future energy systems: a survey," IEEE Transactions on Industrial Informatics, vol. 12, no. 1, pp. 425-436, 2016.

[12] M. M. Rahman, Noor-E-Jannat, M. O. Islam, and M. S. Salakin, "Arduino and GSM based smart energy meter for advanced metering and billing system," in Proc. 2015 International Conference on Electrical Engineering and Information Communication Technology (ICEEICT), Dhaka, Bangladesh, pp. 1-6.

[13] H. Das and L. Saikia, "GSM enabled smart energy meter and automation of home appliances," in Proc. 2015 International Conference on Energy, Power and Environment: Towards Sustainable Growth (ICEPE), Shillong, India, pp. 1-5.

[14] L. Saikia, H. Das, N. D. Choudhury, and T. Malakar, "GPRS enabled smart energy meter with in-home display and application of time of use pricing," in Proc. 2016 IEEE Annual India Conference (INDICON), Bangalore, India, pp. 1-5.

[15] S. Singh, S. L. Arun, and M. P. Selvan, "Regression based approach for measurement of current in single-phase smart energy meter," in Proc. 2017 IEEE Region 10 Symposium (TENSYMP), Cochin, India, pp. 1-5.

[16] V. Paciello, A. Pietrosanto, and P. Sommella, "Smart sensors for demand response," IEEE Sensors Journal, vol. 17, no. 23, pp. 7611-7620, 2017.

[17] J. S. Donnal and S. B. Leeb, "Noncontact power meter," IEEE Sensors Journal, vol. 15, no. 2, pp. 1161-1169, 2015.

[18] Q. Sun, H. Li, Z. Ma, C. Wang, J. Campillo, Q. Zhang, F. Wallin, and J. Guo, "A comprehensive review of smart energy meters in intelligen energy networks," IEEE Internet of Things Journal, vol. 3, no. 4, pp. 464-479, 2016.

[19] L. Labib, M. Billah, G. S. M. Rana, M. N. Sadat, M. G. Kibria, and M. R. Islam, "Design and implementation of low-cost universal smart energy meter with demand side load management," IET Generation, Transmission \& Distribution, vol. 11, no. 16, pp. 3938-3945, 2017.

[20] Y. Xin, C. Mingshuai, L. Xinyang, Z. Hongwei, S. Zongqiang, and L. Liwei, "Research of three-phase high-voltage energy metering device," in Proc. 2017 Chinese Automation Congress (CAC), Jinan, China, pp. 5845-5848.

[21] A. Bernieri, L. Ferrigno, M. Laracca, and A. Rasile, "An AMR-based three-phase current sensor for smart grid applications," IEEE Sensors Journal, vol. 17, no. 23, pp. 7704-7712, 2017.

[22] R. Morello, S. C. Mukhopadhyay, Z. Liu, D. Slomovitz, and S. R. Samantaray, "Advances on sensing technologies for smart cities and power grids: a review," IEEE Sensors Journal, vol. 17, no. 23, pp. 75967610, 2017.

[23] J. Chapa. (2007, Aug) THE ENERGY ORB: Visualize Electricity Consumption!. Accessed: 31 Jan 2019. [Online]. Available: https://inhabitat.com/the-energy-orb-monitor-your-electricity-bill/

[24] S. Rich. (2006, Feb) DIY KYOTO'S WATTSON. Accessed: 31 Jan 2019. [Online]. Available: https://inhabitat.com/diy-kyotos-wattson/

[25] W. N. Stauffer. (2012, Aug) Tomorrow's power grid. Accessed: 31 Jan 2019. [Online]. Available: http://energy.mit.edu/news/tomorrows-powergrid/

[26] V. V. Ranade and J. Beal, "Distributed control for small customer energy demand management," in Proc. 2010 Fourth IEEE International Conference on Self-Adaptive and Self-Organizing Systems, Budapest, pp. $11-20$.

[27] TANGEDCO. (2017, Aug) Revised tariff rates with effect from 11.08.2017 approved rate and payable by the consumer. Accessed: 30 Jan 2019. [Online]. Available: https://www.tangedco.gov.in/linkpdf/ONE_PAGE_STATEMENT.pdf 